\title{
Superdense cosmological dark matter clumps
}

\author{
V. Berezinsky, ${ }^{1,2,3}$ V. Dokuchaev, ${ }^{2,3}$ Yu. Eroshenko, ${ }^{2,3}$ M. Kachelrieß,${ }^{4}$ and M. Aa. Solberg ${ }^{4}$ \\ ${ }^{1}$ INFN, Laboratori Nazionali del Gran Sasso, I-67010 Assergi (AQ), Italy \\ ${ }^{2}$ Center for Astroparticle Physics at LNGS (CFA), I-67010 Assergi (AQ), Italy \\ ${ }^{3}$ Institute for Nuclear Research of the Russian Academy of Sciences, Moscow, Russia \\ ${ }^{4}$ Institutt for fysikk, NTNU Trondheim, N-7491 Trondheim, Norway
}

(Dated: February 18, 2010)

\begin{abstract}
The formation and evolution of superdense clumps (or subhalos) is studied. Such clumps of dark matter (DM) can be produced by many mechanisms, most notably by spiky features in the spectrum of inflationary perturbations and by cosmological phase transitions. Being produced very early during the radiation dominated epoch, superdense clumps evolve as isolated objects. They do not belong to hierarchical structures for a long time after production, and therefore they are not destroyed by tidal interactions during the formation of larger structures. For DM particles with masses close to the electroweak (EW) mass scale, superdense clumps evolve towards a powerlaw density profile $\rho(r) \propto r^{-1.8}$ with a central core. Superdense clumps cannot be composed of standard neutralinos, since their annihilations would overproduce the diffuse gamma radiation. If the clumps are constituted of superheavy DM particles and develop a sufficiently large central density, the evolution of their central part can lead to a 'gravithermal catastrophe.' In such a case, the initial density profile turns into an isothermal profile with $\rho \propto r^{-2}$ and a new, much smaller core in the center. Superdense clumps can be observed by gamma radiation from DM annihilations and by gravitational wave detectors, while the production of primordial black holes and cascade nucleosynthesis constrain this scenario.

PACS numbers: 12.60.Jv, 95.35.+d, 95.85.Pw, 98.35.Gi
\end{abstract}

\section{INTRODUCTION}

Gravitationally bound structures in the universe have developed from primordial density fluctuations $\delta(\vec{x}, t)=$ $\delta \rho / \rho$ that in turn were produced at inflation from quantum fluctuations. In the standard approach to inflation, the spectrum of these primordial fluctuations has a nearly scale-invariant form, $P(k) \equiv \delta_{k}^{2} \propto k^{n_{p}}$ with $n_{p} \simeq 1$. During the radiation-dominated $(\mathrm{RD})$ epoch fluctuations grow slowly, $\delta_{k} \propto \ln \left(t / t_{i}\right)$, while they grow as $\delta_{k} \propto\left(t / t_{\mathrm{eq}}\right)^{2 / 3}$ after the transition to the matterdominated (MD) epoch at $t=t_{\text {eq }}$. Gravitationally bound objects are formed and detach from the cosmological expansion, when fluctuations enter the non-linear regime $\delta \geq 1$. The non-linear stage of fluctuation growth has been studied both analytically $[1-4]$ and in numerical simulations [5-9] for the formation of galaxies and structures on larger scales. The density profile in the inner part of dark matter $(\mathrm{DM})$ halos is given by $\rho(r) \propto r^{-\beta}$, with $\beta \approx 1.7-1.9$ in analytic calculations $[3], \underline{\beta}=1$ in the simulations of Navarro, Frank and White [6] and $\beta=1.5$ in the simulations of Moore et al. [8] and Jing and Suto [9].

The smallest DM objects in the universe, which we shall call clumps or subhalos, are produced first. The evolution of DM clumps has been studied in Ref. [10] in the hierarchical model in which due to the merging of objects a small clump is hosted by a bigger one, the latter is submerged into an even bigger one, etc. The important observation of [10] was the role of tidal interactions, which fully disrupt most clumps. The survived clumps can be further destroyed in the Galaxy by tidal interac- tions in the Galactic plane, near the Galactic center, and in collisions with stars in the halo (see [11] for a review). The characteristic feature of these processes of disruption is that the core of a clump survives and thus the gamma signal from DM annihilations in clumps changes only mildly [11]. A statistical approach to the search for galactic small-scale substructures has been recently proposed in [12, 13].

The mass spectrum of DM clumps has a low-mass cutoff $M_{\min }$ due to the leakage of particles from a clump. This mass is strongly model dependent: It depends on the leakage mechanism (free streaming, collisional damping, etc.), on the properties of the DM particles and the resulting decoupling temperature and others. Therefore, the predicted $M_{\min }$ varies for neutralinos in the minimal supersymmetric standard model from $10^{-7}$ to $10^{-5} M_{\odot}[14,15$.

We have described above the standard cosmological scenario for the clumps. In non-standard scenarios the properties of DM clumps can be very different. In Ref. [16], isothermal perturbations in the DM density were considered within the framework of a spherical collapse model. Perturbations collapse in the RD epoch and produce superdense DM objects. Another possibility for the production of superdense clumps is given by a spiky spectrum of perturbations [17 19]. The general idea common to these scenarios is that there exists a spike on top of a scale-invariant power-law spectrum of perturbations which results in the production of dense clumps in a very early cosmological epoch. In this work, we consider in contrast to [16] the formation of clumps at the RD epoch from adiabatic spiky perturbations. The difference to isothermal perturbations is mainly in their evolution dur- 
ing the linear stage: While isothermal perturbations are frozen in, adiabatic fluctuations grow logarithmically.

We include in this and in the accompanying paper [20] a discussion of the detection prospects of stable superheavy DM particles. Since the annihilation signal from the mean distribution of these particles in the halo is far below observational limits, we examine whether there are new effects which improve the detection chances. One such effect follows from the early kinetic decoupling of superheavy DM particles from the thermal plasma. In this case the cutoff mass can be significantly smaller, as e.g. in the case of ultra-cold WIMPs [18], and thus clumps of practically all masses are formed. This opens the door for the formation of light superdense DM clumps at the RD stage. The only necessary condition is the existence of spiky small-scale perturbations.

This article is organized as follows. We determine the initial properties of the DM clumps, first assuming a standard power-law for the initial cosmological perturbations in Sec. II and then a spiky perturbation spectrum in Sec. III] In Sec. IV] we derive constraints on the superdense clump scenario considering primordial black hole production. Then we study the evolution of the density profile of superdense clump in Sec. VI commenting on the case of neutralinos with masses close to the electroweak mass scale in Sec. $\nabla]$ We present finally our conclusions in Sec. VII.

\section{CLUMPS IN THE STANDARD COSMOLOGICAL SCENARIO}

We briefly remind in this section the formation of clumps and their properties assuming a standard powerlaw spectrum of the initial cosmological perturbations. In contrast to the usual approach, we allow here very small masses of the clumps being inspired by the smallness of $M_{\text {min }}$ in the case of superheavy DM (SHDM), where $M_{\text {min }}$ can be of order of SHDM particle mass $m$.

Small clumps form at the MD epoch for $z \gg 1$, i. e. at a time when the effect of the cosmological constant can be still neglected. In the spherical model of the PressSchechter theory [21, 22], the formation of an object occurs at the time $t_{f}$ when the density contrast $\delta\left(M, t_{f}\right)$ reaches $\delta_{c}=3(12 \pi)^{2 / 3} / 20 \simeq 1.686$. The mean density $\bar{\rho}_{\text {int }}$ and the radius $R$ of the collapsing clumps are

$$
\bar{\rho}_{\mathrm{int}}=\kappa \bar{\rho}\left(z_{f}\right)=\kappa \rho_{\mathrm{eq}}\left(\frac{1+z_{f}}{1+z_{\mathrm{eq}}}\right)^{3}=\kappa \rho_{\mathrm{eq}} \frac{\nu^{3} \sigma_{\mathrm{eq}}^{3}(M)}{\delta_{c}^{3}},
$$

and

$$
R=\left(\frac{3 M}{4 \pi \bar{\rho}_{\text {int }}}\right)^{1 / 3}
$$

where $\kappa=18 \pi^{2} \simeq 178$ [22], $\sigma_{\text {eq }}(M)$ is the variance and $\nu=\delta_{\text {eq }} / \sigma_{\text {eq }}(M)$ is the peak height of the density fluctuations at the time $t_{\text {eq }}$ of matter and radiation equality, while $\rho_{\text {eq }}$ is the density at $t_{\text {eq }}$.

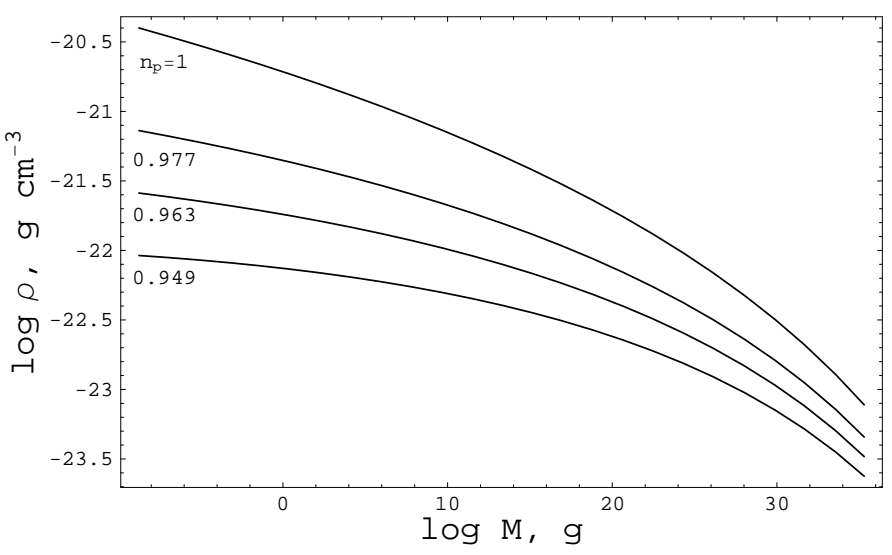

FIG. 1: The mean density $\rho$ of DM clumps as function of the clumps mass $M$ for different spectral indices $n_{p}$ of the primordial density perturbations.

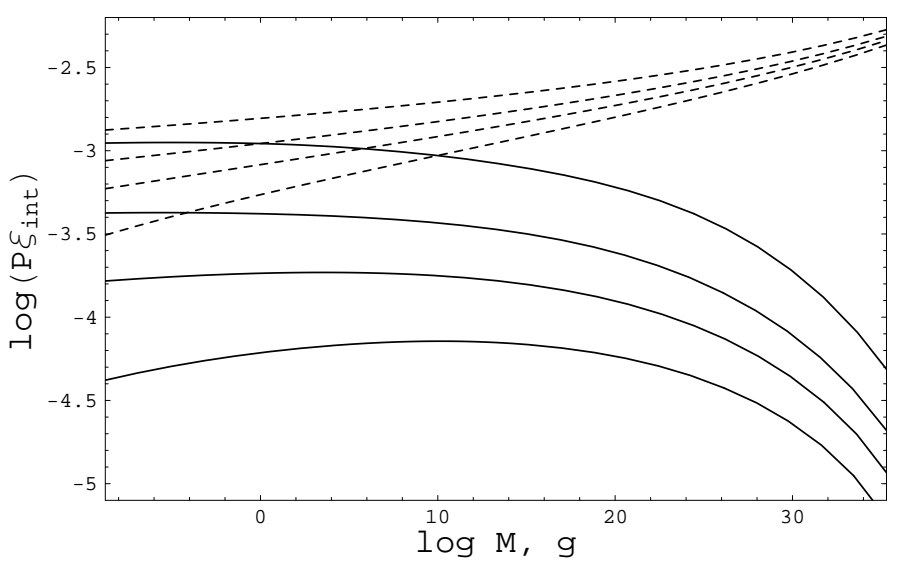

FIG. 2: The fraction of DM in the form of survived clumps per unit logarithmic mass interval $\delta M \sim M$ as function of of clump mass $M$ for $n_{p}=0.949$ (bottom), 0.963, 0.977 and 1 (top): The initial fractions are shown by dashed lines, the present fractions by solid lines.

According to Ref. [11], surviving clumps are characterized by $\nu \simeq 1-3$ and we set $\nu=2$ in all following calculations. Having fixed $\nu$, the dependencies $R(M)$ and $\bar{\rho}(M)$ are unambiguous and the mean density $\bar{\rho}$ of smallscale DM clumps as function of the clumps mass $M$ is shown in Fig. 1.

The mass function of clumps, i.e. the fraction of DM in the form of clumps with mass $M$, is given by [1]

$$
\xi_{\text {int }} \frac{d M}{M} \simeq 0.02(n+3) \frac{d M}{M},
$$

where the effective exponent $n$ in Eq. (3) is found as $n=-3\left(1-2 \partial \log \sigma_{\text {eq }}(M) / \partial \log M\right)$ and depends very weakly on $M$. The simplest inflation models give $P(k) \propto$ $k^{n_{p}}$ with $n_{p} \approx 1$. The 7-year WMAP data, $n_{p}=$ $0.963 \pm 0.014$, favor clearly $n_{s}<1$ [23]. Clumps can 
form nevertheless, because of the presence of additional logarithms in the transfer function. The small-scale spectrum at the epoch of matter-radiation equality can be expressed as [10]

$$
\begin{aligned}
\sigma_{\mathrm{eq}}(M) & \simeq 8.2 \times 10^{3.7\left(n_{p}-1\right)-3}\left(\frac{M}{M_{\odot}}\right)^{\frac{1-n_{p}}{6}} \\
& \times\left[1-0.06 \log \left(\frac{M}{M_{\odot}}\right)\right]^{\frac{3}{2}} .
\end{aligned}
$$

The mass function (3) with the spectrum (4) is shown in Fig. 2 by dashed lines. Its $1 / M$ shape is in good agreement with the corresponding numerical simulations of Ref. [24], only its normalization is a few times smaller than the one found there. For an extrapolation by many orders of magnitudes this must be considered as remarkable agreement.

Note also that using the power-law spectrum that is normalized to the temperature fluctuations of the CMB, i.e. at cosmological scales, for sub-galactic scales or even DM clumps with mass $M \sim 1 \mathrm{~g}$ implies an extrapolation by $\sim 48$ orders of magnitudes. This extrapolation can be justified only within the simplest models for inflation.

Integrating the mass function (3) from $M_{\min }$ to $M \sim$ $10^{2} M_{\odot}$, we obtain the initial (i.e. before possible destruction in the Galaxy) fraction of DM in the form of clumps. In contrast to the standard case of EW scale neutralinos, where $M_{\min } \sim\left(10^{-6}-10^{-8}\right) M_{\odot}$ [15], in superdense clumps the DM particle can have much larger mass and thus $M_{\min }$ can be much smaller. As a result the fraction of surviving clumps increases. In particular for superheavy neutralinos, $M_{\min }$ can be comparable to the particle mass $m$ and the fraction of surviving clumps is calculated as as $0.15,0.18,0.21$ and 0.26 for $n_{p}=0.949$, $0.963,0.977$ and 1 , respectively.

Clumps inside galaxies lose mass and can be destroyed in tidal interactions with stars. The collective gravitational field of the Galactic disk is the most important factor for the clump destruction. A method to study the destruction process of clumps was presented in [15] (for a more detailed approach with gradual mass loss see [1]]), where only clumps with $M>10^{-6} M_{\odot}$ were considered. Here we calculate the survival probability for the wider mass interval $m<M \leq 10^{2} M_{\odot}$, using the same formalism as in [15]. The result for the survival probability $P(\rho)$ at the position of the Sun, $r=8.5 \mathrm{kpc}$ from the Galactic center is presented in Fig. 3. Note that the survival probability $P(\rho)$ means the fraction of surviving clumps near the Sun but most of these clumps have elongated orbits and spend the largest part of their orbital period far from the Sun at the outer parts of the Galactic halo.

The resulting mass function $P \xi_{\text {int }}$ that accounts for the effect of tidal destruction by stars is shown in Fig. 2 by solid lines. Integrating $\int P \xi_{\text {int }} d M / M$ again from $M \sim m$ to $M \sim 10^{2} M_{\odot}$ we obtain the actual fractions of $\mathrm{DM}$ in the form of clumps as $0.006,0.015,0.033$ and 0.085 for $n_{p}=0.949,0.963,0.977$ and 1 , respectively.

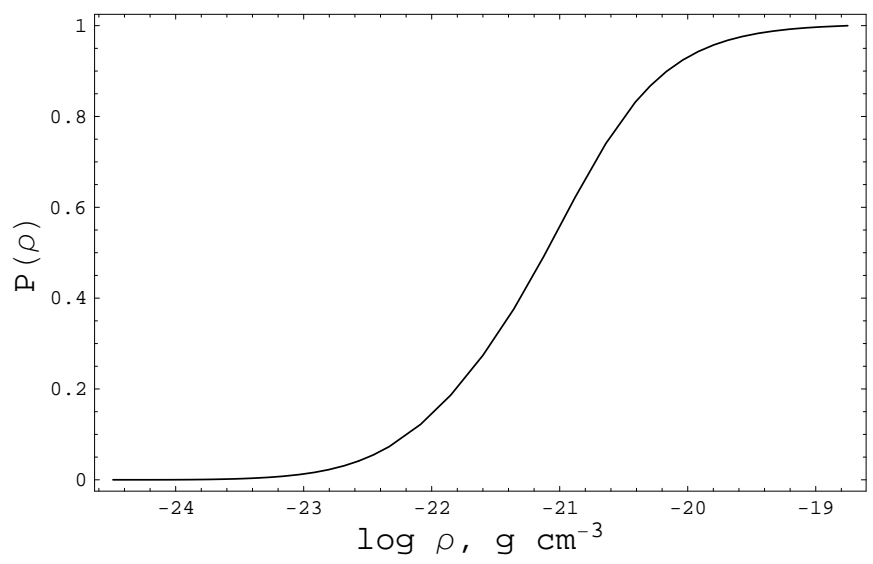

FIG. 3: The survival probability $P(\rho)$ as function of the mean internal clump density $\rho$ at the distance $8.5 \mathrm{kpc}$ from Galactic center.

Clumps formed from the standard power-law spectrum considered above have a rather small density. For many DM particle candidates, including SHDM particles, such clumps are unobservable via their annihilation signal and these clumps can be detected only gravitationally. It has been already suggested that interferometric detectors for gravitational waves like LISA have the capability to detect the tiny variation of the gravitational field, when a compact object crosses the detector. Small SHDM clumps should be included in the list of objects to be searched for by LISA, such as primordial black holes [25], asteroids [26] or compact DM objects of unknown nature [27]. The observable signal is caused by the gravitational tidal force which changes the interferometer arm length and produces correspondingly a phase shift. LISA will have the capability to search for compact objects in the mass interval $10^{16} \mathrm{~g} \leq M \leq 10^{20} \mathrm{~g}$ according to Ref. [25] and $10^{14} \mathrm{~g} \leq M \leq 10^{20} \mathrm{~g}$ according to Ref. [27]. The signal will be in the form of single pulses with its characteristic frequency at the lower end of the expected LISA sensitivity curve and a rate $\sim$ a few per decade, if the objects constitute the major part of DM. The clumps under consideration in this Section present only $1-10 \%$ of all DM, and correspondingly, the detection rate will be 1-2 order of magnitudes lower. In addition, the radii of the clumps generally exceed LISA's arm length $L \simeq 5 \cdot 10^{11} \mathrm{~cm}$ (see the Fig. 4) and the tidal forces will be smaller due to the extension of these objects. Therefore, the detection of the SHDM clumps by LISA seems unlikely. The next generation of gravitational wave interferometers offers more promising perspectives for detection (for details see Ref. 25]). 


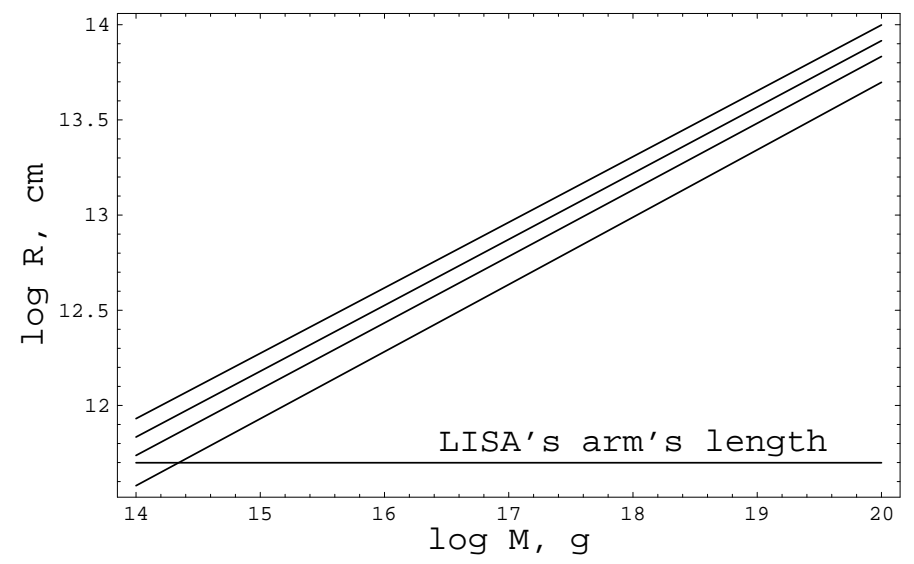

FIG. 4: The mean virial radius $R$ of DM clumps (2) as function of the clump mass $M$ for several values of the spectral index of primordial density perturbations (from top to bottom): $n_{p}=0.949,0.963,0.977$ and 1 . The horizontal line shows LISA's arm's length $L \simeq 5 \cdot 10^{11} \mathrm{~cm}$.

\section{NON-STANDARD PERTURBATIONS AND SUPERDENSE CLUMPS}

\section{A. Spiky density perturbation spectrum}

The variance of the normalized power-law spectrum at the horizon scale during the RD stage was expressed for the standard inflationary scenario in Ref. [28] as

$$
\sigma_{H}(M) \simeq 9.5 \times 10^{-5}\left(\frac{M}{10^{56} \mathrm{~g}}\right)^{\frac{1-n_{p}}{4}} .
$$

We see that in view of current observations $\left(n_{p}<1\right)$ the variance $\sigma_{H}(M)$ is too small for the formation of clumps at the RD stage. Such clumps can be produced effectively only from non-standard spectra containing e.g. spikes.

A sharp peak emerges in the fluctuation spectrum e.g., if an inflationary potential $V(\phi)$ has a flat segment [17, 29]. The mean density perturbation on the horizon scale is $\delta_{\mathrm{H}} \sim M_{\mathrm{Pl}}^{-3} V^{3 / 2} / V^{\prime}$. Hence, if the derivative $V^{\prime}=d V(\phi) / d \phi \rightarrow 0$ for some value of the scalar field $\phi$, then a peak emerges in the perturbation spectrum on the corresponding scale. A similar effect can arise in inflationary models with several scalar fields [30, 31]. In both types of models, the spectrum outside the peak can have an ordinary shape. In particular, it can be a Harrison-Zel'dovich spectrum, and can give rise to galaxies, clusters and superclusters according to the standard scenario.

Another possibility to generate a spiky density perturbation spectrum are cosmological phase transitions, for example the QCD phase transition 32]. If somewhere a high peak arises in the perturbation spectrum, then the corresponding clumps would be the densest DM objects in the universe. Theoretical models for nonstandard spectra were discussed also in [33]. A peak in $P(k)$ was proposed also in [34]. The authors of Ref. [35] found evidence for excess power at small scales $\sim 10 h^{-1} \mathrm{kpc}$ in comparison with a flat primordial power spectrum. This result was obtained from the study of Lyman- $\alpha$ absorbers and can be explained within complex inflation models with the generation of extra power at small scales. Such models can lead to the effective production of very dense clumps.

We will refer to all these models collectively as spiky models or spiky mass-spectrum models.

Dark matter clumps are formed in a wide range of masses, if the power spectrum of primordial cosmological density perturbations has a power-law form. If on the contrary the spectrum has a peak on some scale, then clumps are formed mostly in a narrow range of masses, near the mass that corresponds to this peak.

\section{B. Formation of superdense DM clumps at the RD epoch}

A useful approximation for the nonlinear evolution of perturbations in the radiation dominated epoch is the spherical collapse model [16, 36]. In this model, the evolution of perturbations after the horizon crossing is described by

$$
y(y+1) \frac{d^{2} b}{d y^{2}}+\left[1+\frac{3}{2} y\right] \frac{d b}{d y}+\frac{1}{2}\left[\frac{1+\Phi}{b^{2}}-b\right]=0,
$$

where $y=a(\eta) / a_{\mathrm{eq}}, \eta=d t / d a$ is the conformal time, $a_{\text {eq }}$ is the scale factor at $\eta_{\text {eq }}$, and $\Phi=\delta \rho_{\mathrm{DM}} / \rho_{\mathrm{DM}}$ is the relative overdensity of DM. The radius of the perturbed region is parametrized as

$$
r=a(\eta) b(\eta) \xi
$$

Here, $\xi$ is the comoving coordinate of the spherical layer considered and the value $b(\eta)$ takes into account the slowdown of the cosmological expansion in the perturbed density region. Equation (6) is applicable for the evolution of both entropy and adiabatic perturbations, but has to be used with different initial conditions.

The formation of clumps from entropy perturbations was considered in [16]. In this particular case, the initial data have the form $\Phi=\delta \rho_{\mathrm{DM}} / \rho_{\mathrm{DM}}$ and $d b / d t=0$. The object formed has the density [16]

$$
\rho \simeq 140 \Phi^{3}(\Phi+1) \rho_{\mathrm{eq}} .
$$

For instance, $\Phi \simeq 1 \div 10^{4}$ in the case of axions as DM, and axionic miniclusters have masses in the range $\sim\left(10^{-13} \div\right.$ $0.1) M_{\odot}$. The observational signatures of the presence of these axionic miniclusters in the Galactic halo were considered in [16, 37].

The corresponding method for the nonlinear evolution of adiabatic perturbations during the radiation dominated epoch is described in 38]. For adiabatic perturbations $\Phi=0$, the initial velocity $d b / d t$ is nonzero and 
is defined using linear perturbation theory. The transformation from the Euler description for the growth of density perturbations $\delta$ to the Lagrange description (7) is provided by the relation $b=(1+\delta)^{-1 / 3}$ [36]. The evolution of perturbations with $\delta \ll 1$ on scales less than the horizon is defined by the known analytic solution [32] (see also [38])

$$
\delta=\frac{3 A_{\text {in }}}{2}\left[\ln \left(\frac{x}{\sqrt{3}}\right)+\gamma_{E}-\frac{1}{2}\right] .
$$

In this solution the numerical constant equals $\gamma_{E}-1 / 2 \approx$ $0.077, A_{\text {in }}=\delta_{\mathrm{H}} / \phi, \phi \simeq 0.817, \delta_{\mathrm{H}}$ is the radiation density perturbation on the horizon scale and the variable $x$ is related to the comoving wave-vector $k$ of the perturbation by $x=k \eta$. The connection between $x$ and $y$ is defined by the relation [38]

$$
x=\frac{\pi}{2^{2 / 3}}\left(\frac{3}{2 \pi}\right)^{1 / 6} \frac{c y}{M^{1 / 3} G^{1 / 2} \rho_{\mathrm{eq}}^{1 / 6}} .
$$

It is suitable to connect the analytic solution of the linear theory (9) with the numerical solution of the nonlinear Eq. (6) at the time corresponding to the "transition" value of perturbations with $\delta=0.2$ (see [38]). At this moment we define the initial velocity of the forming DM clump as

$$
\frac{d b}{d y}=-\frac{\delta_{\mathrm{H}} b^{4}}{2 y \phi}
$$

The cosmological expansion of the forming DM clump stops when $d r / d t=0$ or according to Eq. (7) when $d b / d y=-b / y$. The corresponding density and radius of the clump are

$$
\rho_{\max }=\rho_{\text {eq }} y_{\max }^{-3} b_{\max }^{-3}, \quad R_{\max }=\left(\frac{3 M}{4 \pi \rho_{\max }}\right)^{1 / 3},
$$

where $b_{\max }$ and $y_{\max }$ are respectively the values of $b$ and $y$ at the same moment. After decoupling from the cosmological expansion, the object virializes and contracts by a factor two. In Ref. 38] this model was used to describe a noncompact DM object with single mass $\sim 0.1 M_{\odot}$, presumably observable through microlensing. Now we consider the whole possible range of masses and densities of DM clumps. Calculating numerically the solution of Eq. (6) within the above formalism, we find the density of the clump $\rho=\rho\left(M, \delta_{\mathrm{H}}\right)$ as function of its mass $M$ and the radiation perturbation value on the horizon scale $\delta_{\mathrm{H}}$ as shown in Fig. 5 .

Some characteristic values of the clump density $\rho$ are displayed in Fig. 6 for several values of the clump mass $M$. One observes the convergence of curves to $\rho \sim \rho_{\text {eq }} \sim 10^{-19} \mathrm{~g} \mathrm{~cm}^{-3}$ at small $\delta_{\mathrm{H}}$, i.e. for clumps formed near matter-radiation equality. This corresponds to the known analytical results that the evolution during the MD epoch does not depend on the mass but only on the initial (at $t=t_{\text {eq }}$ ) value of the fluctuation.

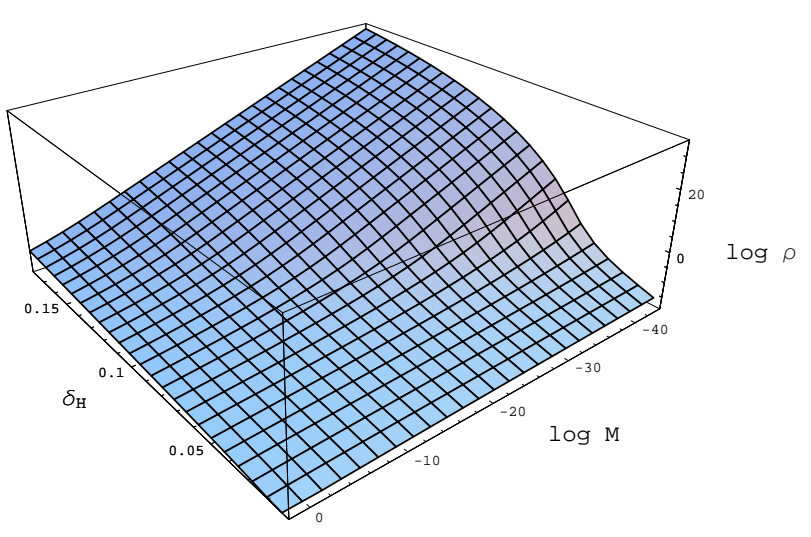

FIG. 5: The mean density $\rho$ (in $\mathrm{g} \mathrm{cm}^{-3}$ ) of DM clumps as function of the perturbation $\delta_{\mathrm{H}}$ in the radiation density on the horizon scale and the clump mass $M$ (in $M_{\odot}$ ).

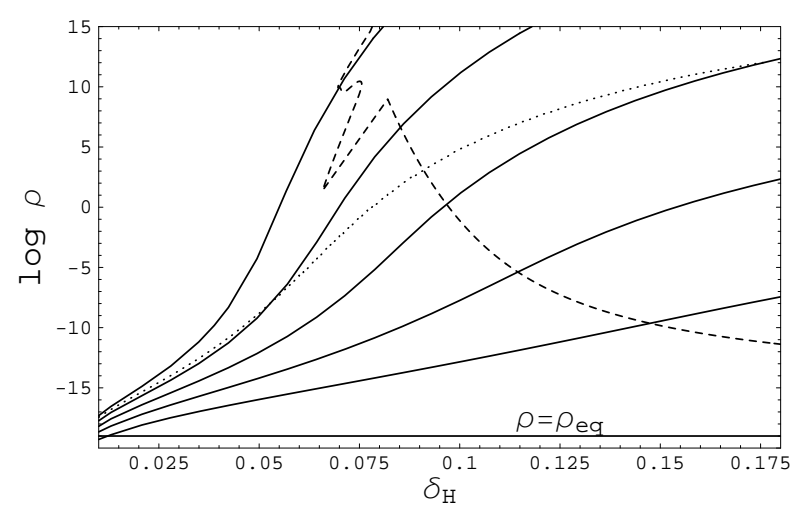

FIG. 6: The mean density $\rho$ (in $\mathrm{g} \mathrm{cm}^{-3}$ ) of DM clumps as function of the perturbation $\delta_{\mathrm{H}}$ in the radiation density on the horizon scale; solid lines from top to bottom are for clump masses $M=10^{-10}, 1,10^{10}, 10^{20}, 10^{30} \mathrm{~g}$. The dashed line is the bound on the clump density from primordial black holes overproduction with threshold $\delta_{c}=0.7$. The time of twobody gravitational relaxation inside the clump core is less than the age of the Universe for clumps above the dotted lines, if the DM particle mass is $m \geq 10^{11} \mathrm{GeV}$.

Note that (in contrast to the case with standard powerlaw spectrum of cosmological perturbations) superdense clumps from a spike in the spectrum are not destroyed by tidal forces and their mass function peaks near a definite mass. Therefore the fraction of DM in the form of such clumps is $\xi \sim 1 / 2$. Half of the volume is in the form of overdensities (clumps), and the remaining space is filled by voids. Because of the compactness of superdense clumps, these clumps can satisfy the condition $R<L \simeq 5 \cdot 10^{11} \mathrm{~cm}$ for the mass interval $10^{14} \mathrm{~g} \leq M \leq 10^{20} \mathrm{~g}$ and are thus observable by the LISA detector. 


\section{CLUMPS AND PRIMORDIAL BLACK HOLES}

The formation of DM clumps leads to several restrictions on the fluctuation spectrum. For instance, highenergy particles from DM annihilations in clumps during the epoch of nucleosynthesis and after it (the cascade nucleosynthesis) might distort the prediction of standard nucleosynthesis.

Another important restriction on the spectrum of the adiabatic perturbations comes from upper limits on the mass and density of primordial black holes (PBHs) [39, 40], because the value of DM density perturbations depends on the radiation density perturbations and the formation of DM clumps can be related to the formation of PBH from the same perturbation spectrum [38]. It should be noted that in the case of entropy perturbations PBHs do not form.

Clumps and PBHs originate from fluctuations of the same type but are formed at different times. The large difference between the masses of DM clumps and of PBH arises because of the large difference in energy density enclosed in a fixed comoving volume as function of time: The energy density of radiation at the RD epoch far exceeds the mass in CDM at the matter domination epoch in the same comoving volume.

The formation of PBHs takes place on the tail of the distribution of Gaussian fluctuations, whereas the main part of clumps is produced from r.m.s. fluctuations. Therefore only a small part of the fluctuations which result in the formation of clumps may produce $\mathrm{PBHs}$ at the $\mathrm{RD}$ epoch. In other words, because of the large threshold of PBH formation, the major part of fluctuations does not collapse into PBHs and evolves continuously up to the end of the RD epoch. During the RD epoch the mass of radiation in the comoving volume varies as $M_{r}(t)=M a\left(t_{\mathrm{eq}}\right) / a(t)$, where the scale-factor of the Universe $a \propto t^{1 / 2}$ and $M$ is the comoving mass at the moment of transition to MD. The mass $M$ equals approximately to the mass of non-relativistic matter inside the fluctuation, i.e. the mass of a clump which may be formed from this fluctuation. On the other hand at horizon crossing $M_{r}(t) \sim 4 \pi(2 c t)^{3} \rho(t) / 3$, where $\rho(t)=3 / 32 \pi G t^{2}$. ¿From these relations for $M_{r}(t)$ we estimate the mass $M_{H}$ and the time $t_{H}$ of $\mathrm{PBH}$ formation as function of the clump mass $M$ as

$$
M_{H} \sim c M^{2 / 3} G^{-1 / 3} t_{\mathrm{eq}}^{1 / 3}, \quad t_{H} \sim G M_{H} / c^{3} .
$$

From the Friedmann equations, the formula for $M_{H}$ was derived exactly in Ref. [38] and is given by

$M_{\mathrm{H}}=\frac{1}{2^{2 / 3}}\left(\frac{3}{2 \pi}\right)^{1 / 6} \frac{M^{2 / 3} c}{G^{1 / 2} \rho_{\mathrm{eq}}^{1 / 6}}=2 \times 10^{5}\left(\frac{M}{0.1 M_{\odot}}\right)^{2 / 3} M_{\odot}$,

while the dependence $t_{H}(M)$ is

$$
t_{\mathrm{H}}=3.7\left(\frac{M}{M_{\odot}}\right)^{2 / 3} \mathrm{~s} .
$$

The fraction of the mass in radiation that is transformed into PBHs at the time $t_{\mathrm{H}}$ is then [40]

$$
\beta=\int_{\delta_{\mathrm{c}}}^{1} \frac{d \delta_{\mathrm{H}}}{\sqrt{2 \pi} \Delta_{\mathrm{H}}} \exp \left(-\frac{\delta_{\mathrm{H}}^{2}}{2 \Delta_{\mathrm{H}}^{2}}\right) \simeq \frac{\Delta_{\mathrm{H}}}{\delta_{\mathrm{c}} \sqrt{2 \pi}} \exp \left(-\frac{\delta_{\mathrm{c}}^{2}}{2 \Delta_{\mathrm{H}}^{2}}\right),
$$

where $\delta_{c}$ is the threshold value of the density perturbations $\delta_{H}$ which result in $\mathrm{PBHs}$ formation. The current PBHs density parameter is $\Omega_{\mathrm{BH}} \simeq \beta a\left(t_{\mathrm{eq}}\right) / a\left(t_{\mathrm{H}}\right)$.

For a large enough value of the r.m.s. perturbation $\Delta_{\mathrm{H}} \equiv\left\langle\delta_{\mathrm{H}}^{2}\right\rangle^{1 / 2}$, an extremely large number of PBHs can be formed [40]. This provides a limitation on $\Delta_{\mathrm{H}}$.

The number density of PBHs depends strictly on the threshold value $\delta_{c}$. In early works, e. g. [40 42], the value of $\delta_{c}=1 / 3$ was obtained. In recent years the phenomenon of critical gravitational collapse was discovered in numerical simulations, for which $\delta_{c} \simeq 0.7$ [43, 44]. Some limits on the number density of PBHs in different mass ranges were obtained in [40, 42]. These restrictions on the value of $\Delta_{\mathrm{H}}$ for PBHs are shown in Fig. 2 for the case $\delta_{c}=0.7$. The relation (14) was used in our calculations. The local minimum on the curve corresponds to the restrictions on the Hawking evaporating PBHs with masses $M_{\mathrm{BH}} \simeq 10^{15} \mathrm{~g}$. For PBHs with a larger mass the only restrictions comes from the condition that their cosmological density parameter $\Omega_{\mathrm{PBH}} \leq 1$.

We recall that PBHs are formed on the tail of the Gaussian perturbation distribution, $\delta_{\mathrm{H}} \geq \delta_{c} \gg \Delta_{\mathrm{H}}$. On the contrary the overwhelming number of DM clumps are formed from the r.m.s perturbations. For this reason in Fig. 2 and in the calculations for DM clumps we put $\delta_{\mathrm{H}} \simeq \Delta_{\mathrm{H}}$.

\section{SUPERDENSE CLUMPS FROM ORDINARY NEUTRALINOS}

We consider in this section the standard case of thermally produced neutralinos with mass close to the electroweak mass scale. We will show that the diffuse gamma flux produced by such neutralinos constituting superdense clumps exceeds the observed flux, and thus superdense clumps should consist of DM particles nonthermally produced.

In order to make our estimate most transparent, we consider first the integral photon flux produced by DM annihilations. This flux is easy to estimate using the annihilation cross section $\langle\sigma v\rangle$ for the process $\chi+\chi \rightarrow$ $\pi^{0}+$ all and the mean density $\bar{\rho}_{\text {int }}$ of neutralinos in a clump.

We calculate first the rate $\dot{N}_{\gamma}$ of gamma-rays with energies higher than $70 \mathrm{MeV}$ produced by a single clump, assuming a $r^{-1.8}$ density profile with core at $r \leq R_{c}$ for a clump with total mass $M$ and radius $R$,

$$
\dot{N}_{\gamma}=1.6 \eta_{\pi^{0}} \frac{\langle\sigma v\rangle}{m_{\chi}^{2}} \bar{\rho}_{\text {int }} M\left(\frac{R}{R_{c}}\right)^{0.6},
$$


where $\eta_{\pi^{0}}$ is neutral pion multiplicity, $R_{c}=x_{c} R$ is the core radius, and $m_{\chi}$ is the neutralino mass.

The total diffuse flux produced in a galactic DM halo can be calculated as

$$
J_{\gamma}^{\mathrm{tot}}=\frac{1}{4 \pi} \bar{n}_{\mathrm{cl}} R_{h} \dot{N}_{\gamma}
$$

with $R_{h}$ as the radius of the DM halo and $\bar{n}_{\mathrm{cl}}$ as the clump mean space density, which is given by the fraction $\xi$ of DM in the form of clumps and the mass $M_{h}$ of the galactic DM halo as

$$
\bar{n}_{\mathrm{cl}}=\frac{3}{4 \pi} \frac{\xi M_{h}}{M} \frac{1}{R_{h}^{3}} .
$$

Using Eqs. (17,[19) we can express the diffuse flux $J_{\gamma}^{\text {tot }}$ in terms of the mean density of neutralinos in clumps $\bar{\rho}_{\text {int }}$, the main characteristic of superdense clumps:

$$
J_{\gamma}^{\mathrm{tot}}=f_{\mathrm{NFW}} \frac{0.4}{\pi} \frac{\eta_{\pi^{0}} \xi R_{h}}{x_{c}^{0.6}} \frac{\bar{\rho}_{\text {int }} \bar{\rho}_{\text {halo }}^{D M}}{m_{\chi}^{2}}\langle\sigma v\rangle,
$$

where $\bar{\rho}_{\text {halo }}^{D M}$ is the mean density of DM halo. To take into account the NFW density profile one must multiply the homogeneous halo result by the additional factor $f_{\mathrm{NFW}}=$ 293. The obtained flux is given as convenient expression where most of parameters are observationally known and the main characteristic of superheavy clumps is $\bar{\rho}_{\text {int }}$. For the parameters in Eq. (20), we use $\eta_{\pi^{0}}=10$ appropriate for gauge boson decays, $\xi=1 / 2$ (for spiky scenario), $R_{h} \approx 200 \mathrm{kpc}$ and $\bar{\rho}_{\text {halo }}^{D M}=1.1 \times 10^{-3} \mathrm{GeV} / \mathrm{cm}^{3}$ obtained as $3 M_{h} / 4 \pi R_{h}^{3}$. We assume $x_{c} \approx 0.1$.

Typically superdense clumps have very large densities (see Fig. 6) and for ordinary neutralinos the resulting gamma-ray flux exceeds the observations. First we analyze the problem, whether ordinary neutralinos are compatible with a spiky scenario of clump production. With this aim we choose in Eq. (20) parameters which minimize the flux. For the mean density of neutralinos in a clump, $\bar{\rho}_{\text {int }}$, we take the minimum value, assuming neutralino produced at the beginning of the MD epoch. In this case $\bar{\rho}_{\text {int }}=178 \rho_{e q}=8.3 \times 10^{6} \mathrm{GeV} / \mathrm{cm}^{3}$ (see Eq. (1) in the limit $z_{f} \rightarrow z_{\text {eq }}$ ). We parametrize the annihilation cross-section $\langle\sigma v\rangle$ by the characteristic value $1 \times 10^{-26} \mathrm{~cm}^{3} / \mathrm{s}$ as

$$
\langle\sigma v\rangle_{26}=\langle\sigma v\rangle /\left(10^{-26} \mathrm{~cm}^{3} \mathrm{~s}^{-1}\right) .
$$

With this parameters the minimum gamma-ray flux is

$$
J_{\gamma}^{\text {tot }}=4.3\langle\sigma v\rangle_{26} m_{100}^{-2} \quad \mathrm{~cm}^{-2} \mathrm{~s}^{-1} \mathrm{sr}^{-1},
$$

where $m_{100}$ is the neutralino mass $m_{\chi}$ in units of 100 $\mathrm{GeV}$.

The integral flux (22) is about 5 orders of magnitudes larger than the observed flux. Does it help to increase the neutralino mass or to consider a smaller annihilation cross section? To answer the first part of this question, we consider now the differential isotropic diffuse photon flux observed by Fermi-LAT at $|b|>60$ degrees [50],

$$
J_{\mathrm{obs}}(E)=6 \times 10^{-7}\left(\frac{E}{\mathrm{GeV}}\right)^{-2.45} \mathrm{GeV}^{-1} \mathrm{~cm}^{-2} \mathrm{~s}^{-1} \mathrm{sr}^{-1}
$$

The differential photon flux produced by annihilations can be obtained from Eq. (20) replacing $2 \eta_{\pi^{0}}$ by $d N /\left(2 m_{\chi} d x\right)$, where $d N / d x$ is the number of photons with energy $E=x m_{\chi} / 2$ produced per annihilation. Since moreover $d N / d x$ increases at small $x$ for increasing $m_{\chi}$, the ratio $J_{\gamma}(E) / J_{\text {obs }}(E)$ is practically constant. The minimal allowed annihilation cross-section of neutralinos obtained in [4]] for the case of strongly suppressed $s$-wave annihilations is $\langle\sigma v\rangle=1.7 \times 10^{-30} m_{100}^{-2} \mathrm{~cm}^{3} / \mathrm{s}$. With these parameters the minimum gamma-ray flux is still above the measured Fermi-LAT flux. Finally, we remark that even postulating at tree-level only couplings e.g. to electrons would lead to an overproduction of photons via Bremsstrahlung, c.f. e.g. Ref. [51]. In conclusion, ordinary neutralino as other thermally produced DM particles are excluded as constituents of superdense clumps. A DM particle suitable to compose superdense clumps must have a smaller annihilation cross section than allowed for a thermal relic.

\section{DENSITY PROFILE EVOLUTION}

In the case of a spiky spectrum, clumps are formed not in the process of hierarchical clustering but due to the evolution of isolated density fluctuations. Such a scenario is similar to the analytic approach in Ref. [3]. The ordinary gravitational contraction combined with the multistream instability produces the universal power-law density profile with exponent $\beta=1.7-1.9$ [3] . This powerlaw shape for $\rho(r)$ has been recently confirmed in the numerical simulations [45] for neutralino clump formation during the MD epoch. We assume here that the clumps produced at the RD stage in the process of ordinary gravitational contraction have a profile $\rho(r) \propto r^{-1.8}$ for $R_{c}<r<R$ and $\rho(r)=\rho_{c}=$ const for $r<R_{c}$, where $R_{c}$ is the unknown core radius of the clump. This core may be produced due to tidal forces [10] in the clumps formed at the MD stage. In this case a large core is produced with $x_{c} \equiv R_{c} / R \sim 0.01-0.1$. More precisely, the given value corresponds not to the radius of the constantdensity core but to the break in the slope of the density profile. Moreover, the above-mentioned calculations are valid for the MD dominated epoch, where the process of core formation can be much different from that at the RD epoch.

Another estimate for the core size has been obtained in Ref. [3], where $x_{c}$ is defined by the damping mode of the perturbations. The authors obtained $x_{c} \sim \delta_{\text {eq }}^{3}$, where $\delta_{\text {eq }}$ is the value of density fluctuation at the beginning of the MD stage. However, this estimate is also valid for the MD epoch. 
At the current level of knowledge, the relative radius $x_{c} \equiv R_{c} / R$ of the core produced by ordinary gravitational contraction must be considered as a free parameter. In the most conservative case we use $x_{c} \sim 0.1$. The central density $\rho_{c}$ depends on the mean clump density $\bar{\rho}=8 \rho_{\max }\left(\right.$ see (12)) as $\rho_{c}=\bar{\rho} /\left(3 x_{c}^{2}\right)$.

We shall briefly discuss the evolution of superdense clumps formed from superheavy particles. Quantitatively, it will be considered in the accompanying paper II.

The first stage of evolution, the ordinary gravitational contraction, proceeds like in the case of ordinary neutralinos and results in the production of a $\rho(r) \sim r^{-1.8}$ profile with a relatively large core, $x_{c} \sim 0.01-0.1$. Other processes can become important at the second stage: (i) two-body gravitational scattering and (ii) some limiting effect like Fermi degeneracy or the intensive annihilation of particles. In the cores of superdense clumps with large densities $n$ of particles the binary gravitational scattering of constituent DM particles with large masses $m$ may become the dominant process, which causes the "gravithermal instability" or "gravithermal catastrophe", well known in theory of globular star clusters. Note that this effect takes place only for superheavy DM and only in the most dense parts of superdense clumps. In Fig. 6, this region is located above the dotted line.

How can it be that gravitational two-body scattering becomes the dominant process? It occurs because gravitational scattering is proportional to $m^{2}$, while EW scattering of these particles is proportional to $1 / \mathrm{m}^{2}$. The other two factors are the large density $n$ of particles in the core and the long-range character of gravitational interactions. All this provides the fast gravitational relaxation of the system. As a result of the gravithermal instability a clump develops an isothermal density profile $\rho(r) \propto r^{-2}$ with a tiny core. This core can be produced by the pressure of a degenerate gas in the case of superheavy fermionic particles or by the inverse flow due to the annihilation of particles in the clump center [48, 49]. In these cases the radius of the new core is determined by the elementary particle properties of dark matter.

\section{CONCLUSIONS}

Superdense clumps can be produced from isothermal perturbations [16] or from spikes in the spectrum of adiabatic perturbations [17, 38]. These objects are produced in the very early universe during the $\mathrm{RD}$ epoch. In princi- ple, the perturbation spectrum may include both a scaleinvariant power-law component and spikes. Being produced very early during the radiation dominated epoch, superdense clumps evolve as isolated objects. They do not belong to hierarchical structures for a long time after production, and therefore they are not destroyed by tidal interactions during the formation of large-scale structures.

In the case of EW scale mass particles, e.g. ordinary neutralinos, the density profile has a $r^{-1.8}$ shape with a relatively large core characterized by $R_{c} / R \sim 0.01-0.1$, produced by tidal forces. Ordinary neutralinos are excluded as the constituents of superdense clumps, because they overproduce the diffuse gamma-ray spectrum above $100 \mathrm{MeV}$. The constituent DM particles in superdense clumps must be either very weakly annihilating or be superheavy, or both. The limit on the superdense clumps is imposed by primordial black holes which originated from the same perturbation spectrum. The allowed intrinsic densities of superdense clumps are shown in Fig. 6. The formation of superdense clumps at the RD epoch was studied previously using somewhat different assumptions in Refs. [16, 38, [53].

The density profile in superdense clumps depends on the properties of the DM particles. For very heavy constituent particles and large intrinsic densities of the clumps a "gravithermal catastrophe" (instability) may develop in superdense clumps. As a result the initial density profile turns into an isothermal one, $\rho_{\text {int }}(r) \propto 1 / r^{2}$, and the large initial core collapses into a tiny, very dense new core. The steep density profile and the smallness of the core lead to a strong DM annihilation signal. The radiation produced by DM annihilations restricts this scenario, e.g. due to the cascade nucleosynthesis following standard nucleosynthesis. On the positive side, superdense clumps can lead to detectable gamma radiation even in the case of superheavy DM particles [20]. Superdense clumps can be in principle observed also by gravitational wave detectors.

\section{Acknowledgments}

VB is grateful to the A. Salam International Centre for Theoretical Physics for hospitality during the work on this paper and to A. Smirnov for valuable discussions. This work was supported by the Russian Federal Agency for Science and Innovation under state contract 02.740.11.5092 and by the grants of the Leading scientific school 959.2008.2 and 438.2008.2.
[1] J.E. Gunn, Astrophys. J. 218, 592 (1977).

[2] E. Bertschinger, Astrophys. J. Supp. 58, 39 (1985).

[3] A. V. Gurevich and K. P. Zybin, Sov. Phys. JETP 67, 1 (1988); Sov. Phys. JETP 67, 1957 (1988); Sov. Phys.
Usp. 165, 723 (1995).

[4] P. Sikivie, I.I. Tkachev and Y. Wang, Phys. Rev. D 56, 1863 (1997) arXiv:astro-ph/9609022 v1].

[5] J.R. Gott, Astrophys. J. 201, 296 (1975) 
[6] J. F. Navarro, C. S. Frenk and S. D. M. White, Astrophys. J. 462, 563 (1996) arXiv:astro-ph/9508025.

[7] T. Fukushige, J. Makino, Astrophys. J. Lett. 477, 9 (1997).

[8] B. Moore et al., Astrophys. J. Lett., 524, 19 (1999).

[9] Y. P. Jing and Y. Suto, Astrophys. J. 529, L69 (2000) arXiv:astro-ph/9909478.

[10] V. Berezinsky, V. Dokuchaev and Y. Eroshenko, Phys. Rev. D 68, 103003 (2003) arXiv:astro-ph/0301551.

[11] V. Berezinsky, V. Dokuchaev and Y. Eroshenko, Phys. Rev. D 77, 083519 (2008) arXiv:0712.3499 [astro-ph]].

[12] M. Kamionkowski and S. M. Koushiappas, Phys. Rev. D 77, 103509 (2008) arXiv:0801.3269 v2 [astro-ph]].

[13] M. Kamionkowski, S.M. Koushiappas and M. Kuhlen, arXiv:1001.3144v2 [astro-ph.GA]].

[14] C. Schmid, D. J. Schwarz and P. Widerin, Phys. Rev. D 59, 043517 (1999) arXiv:astro-ph/9807257; A. M. Green, S. Hofmann and D. J. Schwarz, JCAP 0508, 003 (2005) arXiv:astro-ph/0503387; E. Bertschinger, Phys. Rev. D 74, 063509 (2006) arXiv:astro-ph/0607319.

[15] V. Berezinsky, V. Dokuchaev and Yu. Eroshenko, Phys. Rev. D 73, 063504 (2006) arXiv:astro-ph/0511494.

[16] E. W. Kolb and I. I. Tkachev, Phys. Rev. D 50, 769 (1994) arXiv:astro-ph/9403011.

[17] A. A. Starobinsky, JETP Lett. 55, 489 (1992) [Pisma Zh. Eksp. Teor. Fiz. 55, 477 (1992)].

[18] G. B. Gelmini and P. Gondolo, JCAP 0810, 002 (2008) arXiv:0803.2349 [astro-ph]].

[19] P. Scott and S. Sivertsson, arXiv:0908.4082 [astroph.CO].

[20] V. Berezinsky, V. Dokuchaev, Yu. Eroshenko, M. Kachelrieß and M. Aa. Solberg, arXiv:1002.3445 [astro-ph.GA]] (Paper II).

[21] W. H. Press and P. Schechter, Astrophys. J. 187, 425 (1974).

[22] C. Lacey, S. Cole, Mon. Not. R. Astron. Soc. 262, 627 (1993).

[23] D. Larson et al. [WMAP Collaboration], arXiv:1001.4635v1 [astro-ph.CO].

[24] J. Diemand, B. Moore and J. Stadel, Nature 433, 389 (2005) arXiv:astro-ph/0501589.

[25] N. Seto and A. Cooray, Phys. Rev. D 70, 063512 (2004) arXiv:astro-ph/0405216v1]

[26] P. Tricarico, Class. Quantum Grav. 26, 085003 (2009).

[27] A. W. Adams and J. S. Bloom, arXiv:astro-ph/0405266v2.

[28] A. M. Green and A. R. Liddle, Phys. Rev. D 56, 6166 (1997) arXiv:astro-ph/9704251.

[29] P. Ivanov, P. Naselsky and I. Novikov, Phys. Rev. D 50, 7173 (1994).

[30] J. Yokoyama, Astron. Astrophys. 318, 673 (1997)
arXiv:astro-ph/9509027.

[31] J. Garcia-Bellido, A. D. Linde and D. Wands, Phys. Rev. D 54, 6040 (1996) arXiv:astro-ph/9605094.

[32] C. Schmid, D. J. Schwarz and P. Widerin, Phys. Rev. D 59, 043517 (1999) arXiv:astro-ph/9807257.

[33] J. M. Cline, P. Crotty and J. Lesgourgues, JCAP 0309 , 010 (2003) arXiv:astro-ph/0304558.

[34] D. J. H. Chung, E. W. Kolb, A. Riotto and I. I. Tkachev, Phys. Rev. D 62, 043508 (2000) arXiv:hep-ph/9910437.

[35] M. Demianski and A. G. Doroshkevich, Astrophys. J. 597, 81 (2003) arXiv:astro-ph/0304484.

[36] T. Padmanabhan and K. Subramanian, Astrophys. J. 417, 3 (1993).

[37] E. W. Kolb and I. I. Tkachev, Astrophys. J. 460, L25 (1996) arXiv:astro-ph/9510043.

[38] V. I. Dokuchaev and Yu. N. Eroshenko, J. Exp. Theor. Phys. 94, 5 (2002) [Zh. Eksp. Teor. Fiz. 94, 5 (2002)] arXiv:astro-ph/0202021.

[39] Ya. B. Zel'dovich and I. D. Novikov, Sov. Astron. 10, 602 (1967).

[40] B. J. Carr, Astrophys. J. 201, 1 (1975).

[41] D. K. Nadezhin, I. D. Novikov, and A. G. Polnarev, Sov. Astron. J. 55, 216 (1978).

[42] I. D. Novikov, A. G. Polnarev, A. A. Starobinsky, and Ya. B. Zeldovich, Astron. Astrophys. 80, 104 (1979).

[43] M. W. Choptuik, Phys. Rev. Lett. 70, 9 (1993).

[44] J. C. Niemeyer, Proceedings of Dark Matter 98, Los Angeles (ed. D. Cline); astro-ph/9806043.

[45] B. Moore, J. Diemand, J. Stadel and T. Quinn, Nature 433389 (2005).

[46] L. Spitzer and W.C. Saslaw, Astrophys. J. 143, 400 (1966).

[47] C. S. Kochanek and M. J. White, Astrophys. J. 543, 514 (2000) arXiv:astro-ph/0003483.

[48] V. S. Berezinsky, A. V. Gurevich and K. P. Zybin, Phys. Lett. B 294, 221 (1992).

[49] V. Berezinsky, A. Bottino and G. Mignola, Phys. Lett. B 391, 355 (1997) arXiv:astro-ph/9610060.

[50] Markus Ackermann, talk at TeV Particle Astrophysics 2009, slides available at http://www-conf.slac.stanford.edu/tevpa09/Talks.asp

[51] L. Bergstrom, T. Bringmann, M. Eriksson and M. Gustafsson, Phys. Rev. Lett. 95, 241301 (2005) arXiv:hep-ph/0507229]; M. Kachelrieß, P. D. Serpico and M. Aa. Solberg, Phys. Rev. D 80, 123533 (2009) arXiv:0911.0001 [hep-ph]].

[52] V. Berezinsky, A. Bottino, J. Ellis, N. Fornengo, G. Mignola, S. Scopel, Astrop. Phys. 5, 1 (1996).

[53] M. Ricotti, A. Gould, Astrophys. J. 707, 979 (2009) arXiv:0908.0735 v2 [astro-ph.CO]]. 